\title{
Biomarcadores no envolvimento do sistema nervoso central no Lúpus Eritematoso Sistêmico
}

\author{
Barbara F. de Melo* Padovan; Mariana de B. Tozarini*; Talita R. Marques*; Fernando A. Peres; Simone \\ Appenzeller.
}

\section{Resumo}

Objetivos:Analisar a prevalência de distúrbio olfatório em pacientes com LES, correlacionando com a presença de MNP, níveis de anti-P e atividade e dano da doença. Métodos:Foram incluídos pacientes com diagnóstico de LES, seguidos no ambulatório de reumatologia do HC-UNICAMP. Resultados:Alterações olfatórias estavam presentes em 62 (51,6\%) pacientes e em $12(24 \%)$ controles $(p=0,003)$. Anti-P positivo foi associado com psicose $(p=0,046)$. Atrofia de amígdala esquerda foi associada com LDI $(p=0,02)$.

\section{Palavras-chave \\ Anticorpo Anti-P Ribossomal; LES; Sistema Nervoso Central}

\section{Introdução}

Lúpus Eritematoso Sistêmico (LES) é uma doença autoimune, de etiologia parcialmente desconhecida e Acomete principalmente mulheres na proporção de 10 para cada homem.

Manifestações neuropsiquiátricas (MNP) no LES acometem de 12 a $95 \%$ dos pacientes e o acometimento está relacionado a ação de autoanticorpos, dentre outros. $\mathrm{O}$ anti-P é um autoanticorpo altamente especifico para o LES e está associado a apoptose celular e neurodegeneração de estruturas límbicas (amígdala e hipocampo) que são relacionadas com emoções, memória e olfato.

Alterações no olfato são associadas a doenças psiquiátricas e neurológicas como Alzheimer, esquizofrenia, depressão e doença de Parkinson desde a década de 70 . No LES a alteração do olfato é associada com o índice de dano da doença e com histórico de MNP.

O objetivo deste trabalho foi analisar a prevalência de distúrbio olfatório em pacientes com LES, correlacionando com a presença de MNP, níveis de anti$\mathrm{P}$ e atividade e dano da doença.

\section{Resultados e Discussão}

Foram incluídos 120 pacientes (93,3\% mulheres; média de idade 40,3 anos; $\mathrm{DP} \pm 11,3$ anos) e 50 voluntários sadios (89,3\% mulheres; média de idade 39,4 anos; $\mathrm{DP} \pm 14,7$ anos). Anti-P foi encontrado exclusivamente em pacientes e estava presente em 13 (10,8\%) deles $(p<0,001)$. O anti-P não foi associado com a presença de MNP $(p=0,730)$, porém quando analisamos os subitens classificatórios de MNP separadamente, observamos associação entre anti-P positivo e psicose $(p=0,046)$. Não encontramos associações entre alteração olfatória e outras manifestações clínicas. Alterações olfatórias estavam presentes em $62(51,6 \%)$ pacientes e em 12 $(24 \%)$ controles $(p=0,003)$. A alteração olfatória foi inversamente correlacionada com ansiedade $(p=0,004$, $R=-0,18)$, depressão $(p=0,01, R=-0,232)$, dano cumulativo $(p=0,002, R=-0,282)$ e idade $(p<0,001, R=-$ $0,353)$ nos pacientes.
Atrofia de amígdala direita foi encontrada em 15 pacientes (12,5\%) e atrofia de amígdala esquerda em 24 $(20 \%)$ pacientes. Todos pacientes com atrofia de amígdala direita apresentavam atrofia bilateral de amígdala.

Atrofia de amígdala esquerda foi associada com escores de LDI $(p=0,024)$, sendo que pacientes com atrofia tinham média de $27,04 \pm 5,48$ pontos no LDI enquanto que pacientes sem atrofia tinham média de 29,69 $\pm 4,88$ pontos no LDI. Atrofia de amígdala direita não se associou com escores do LDI $(p=0,104)$, mas associouse com a fase de discriminação de odores $(p=0,006)$ do teste de avaliação olfatória.

A atrofia de amígdala direita e esquerda não foi associada com a presença do anti-P $(p=0,653$ e $p=0,707$, respectivamente). Não observamos outras associações ou correlações.

\section{Conclusões}

Pacientes com LES apresentam uma diminuição significativa do olfato quando comparados a controles sadios. Alterações olfatórias são associadas com ansiedade, depressão, dano cumulativo e idade e podem ser uma ferramenta útil e de fácil utilização para o diagnóstico precoce do envolvimento do SNC em doenças autoimunes. Anti-P é altamente específico para o LES e pode estar envolvido no mecanismo de atrofia das estruturas do sistema límbico.

\section{Agradecimentos}

FAPESP (2008/02917-0; 2011/15422-2; 2014/00734-7) CNPq: (471343/2011; 300447/2009-4; 473328/2013-5)

Appenzeller S, Cendes F, Costallat LT. Arthritis Rheum. 2009:61;680-687. Rahman A, Isenberg DA. N Engl J Med. 2008;358:929-939.

Bonfa E, Golombek SJ, Kaufman LD, Skelly S, Weissabach H, Brot N, Elkon KB. N Engl J Med. 1987;317:265-271.

Strous RD, Shoenfeld Y. Autoimmun Rev. 2006;6:54-60.

Shoenfeld N, Agmon-Levin N, Flitman-Katzevman I, Paran D, Katz BS, Kivity S, Langevitz P, Zandman-Goddard G, Shoenfeld Y. Arthritis Rheum 2009; 60:1484-1487. 